\title{
Influence of Muscle Fatigue on Three-Dimensional Scapular Kinematics During Repeated Arm Elevation
}

\author{
Hitoshi Koda*1, Yoshihiro Kai ${ }^{2}$, Noriyuki Kida ${ }^{3}$, Shin Murata ${ }^{2}$, Yuichiro Miura ${ }^{4}$, Hideaki Fukushima ${ }^{4}$ and Toru \\ Morihara $^{5}$
}

${ }^{1}$ Department of Rehabilitation, Faculty of Health Science, Kansai University of Welfare Sciences, Japan

${ }^{2}$ Department of Physical Therapy, Faculty of Health Science, Kyoto Tachibana University, Japan

${ }^{3}$ Graduate School of Science and Technology, Kyoto Institute of Technology, Japan

${ }^{4}$ Rehabilitation Unit, Fushimi Okamoto Hospital, Japan

${ }^{5}$ Department of Orthopedics, Graduate School of Medical Science, Kyoto Prefectural University of Medicine, Japan

Received: 制: Octobet 27, 2018; Published: 制: November 09, 2018

*Corresponding author: Hitoshi Koda, Department of Rehabilitation, Faculty of Health Science, Kansai University of Welfare Sciences, Japan

\begin{abstract}
Previous studies have reported that shoulder fatigue leads to scapular dyskinesia and increased mechanical stress. However, none reported the effect of fatigue resistance. To investigate the effect of fatigue resistance on scapular kinematics during repeated arm elevation. Seventeen healthy participants carried out repeated arm elevation with a dumbbell in the scapular plane at the elbow extension position. Using an electromagnetic tracking device, the angle of the scapular upward rotation, scapular posterior tilting, and scapular external rotation were measured. Arm elevation data were interpolated into 100 frames; the first elevation ( $0 \%), 25 \%$ of iteration count, 50\%, 75\%, and the final elevation (100\%) were analyzed. There was a significant increase in the scapular upward rotation angle between the $100 \%$ and $0 \%$ iteration count, and a decrease in the scapular posterior tilting angle at $75 \%$ and $100 \%$, compared with $0 \%$. There was no significant effect of the condition on the scapular external rotation angle. Muscle fatigue by repeated arm elevation increased the upward rotation angle, which was maintained until just before complete exhaustion. The decrease in the posterior tilting angle was observed before (when the iteration count exceeded $50 \%$ ) the increase in the upward rotation angle

Keywords: Scapular Dyskinesia; Arm Elevation; Repeated Movement; Fatigue Resistance
\end{abstract}

\section{Introduction}

The shoulder joint contributes towards the movement of the upper limbs; however, it has poor stability and is easily prone to injury [1]. Shoulder injuries are common in overhead athletes, with as many as $44 \%$ experiencing shoulder problems and $29 \%$ having shoulder pain at some point in their career [2]. The high prevalence of shoulder pain in these athletes is attributed largely to repetitive and excessive stress placed on the shoulder. In this year's World Baseball Classic, repetitive stress due to more pitching was regarded as a problem and introduced the pitcher use limitation rules.

Scapular dyskinesia is one of the factors to increase mechanical stress in the shoulder joint [3-5]. Willmore et al. [5] showed that a decrease in scapula upward rotation and posterior tilting occur when there is an increase in the compressing and shearing force under the surface of the acromion. Sanchez et al. [6] found that the ratio of scapular dyskinesia in the shoulder pain group was higher than that in the pain-free group. The reported scapular dyskinesia is related to an increase in mechanical stress or shoulder injuries.

Scapular dyskinesia is caused by muscle weakness or trunk misalignment $[7,8]$. On the other hand, fatigue of the shoulder muscles due to repetitive and prolonged overhead sports activity also appears to temporarily alter the scapular kinematics [9-13]. Ebaugh et al. [9] found post-fatigue scapular kinematic alterations, with increased scapular upward rotation and clavicular retraction in the mid ranges of arm elevation. Chopp et al. [11] showed decreased scapular posterior tilting and increased scapular external rotation after a fatiguing task. Thus, previous studies reported that shoulder fatigue leads to scapular dyskinesia and increased mechanical stress; however, to our knowledge, none of them researched fatigue resistance, which is how much fatigue leads to scapular dyskinesia. 
The purpose of this study was to investigate the effect of fatigue resistance on scapular kinematics by analyzing the kinematics during repeated arm elevation. We hypothesized that fatigue resistance is different by direction of scapular kinematics.

\section{Materials and Methods}

Seventeen healthy subjects were recruited in this study. Their mean age was $20.5 \pm 0.6$ years, mean height was $169.6 \pm 4.6 \mathrm{~cm}$, and mean body mass was $60.6 \pm 5.9 \mathrm{~kg}$. No subject had any history of shoulder pain or injuries before participating in this study.

The study was performed in accordance with the World Medical Associations Declaration of Helsinki. The purpose, nature, and potential risks of the experiments were fully explained to the participants, and all participants gave written, informed consent prior to their inclusion in the study. The study protocol was approved by the research ethics committees of the respective affiliated institutes (17-3).

Participants held a dumbbell with their right hand and repeated arm elevation as much as possible in the scapular plane at the elbow extension position; movement from the downward position to the maximum elevated position and the opposite movement was repeated during constant 3-s periods. Repeated arm elevation was continued until either the appearance of compensatory movements, the participants could not fulfill the task within the time or were offered retirement.

The dumbell was approximately $15 \%$ of the twice maximum shoulder abduction force. For the measurement of the shoulder abduction force, we used a hand-held dynamometer, Mobie (SAKAImed, Osaka, Japan); force loading was applied with the shoulder at a $90^{\circ}$ elevation in the scapular plane and the elbow extended $0^{\circ}$ with the forearm midway between a thumb-up and thumb-down position. The belt of the Mobie was fixed to the distal humerus and the floor. The participants were asked to exert a maximum isometric contraction in the shoulder abduction direction [14].

Kinematic data were collected during the task using an electromagnetic tracking device, LIBERTY (Polhemus, Vermont, USA), and Motion Monitor software ${ }^{\circledR}$ version 8.43 (Innovative Sports Training., Inc., Chicago, IL). Data were collected at a $120-\mathrm{Hz}$ sampling rate. The global coordinate system was established by a transmitter mounted on a rigid wooden base frame aligned with the cardinal planes of the body. Electromagnetic sensors were attached to the sternum, acromion, and humerus on the right side. The bony landmarks were palpated and digitized while the subject sat on a plastic chair and relaxed the arm to the side of the body. The landmarks were chosen in accordance with the International Society of Biomechanics [15]: the spinous processes of the $7^{\text {th }}$ cervical and $8^{\text {th }}$ thoracic vertebrae, suprasternal notch, and xiphoid process were the thoracic landmarks, the glenohumeral joint rotation center (estimated by the rotation method) and medial and lateral epicondyles were the humeral landmarks, and the acromial angle, root of the spine of the scapula, and the inferior angle were the scapular landmarks. Measurement angles were the scapular upward rotation, scapular posterior tilting, scapular external rotation, arm elevation, and horizontal adduction between the humeral and scapula plane.

We chose five sets of elevation data: the first elevation $(0 \%)$, $25 \%$ of the iteration count (25\%), $50 \%$ of the iteration count (50\%), $75 \%$ of the iteration count (75\%), and the final elevation (100\%). Each arm elevation data was interpolated into 100 frames using the spline function. Scapular upward rotation, posterior tilting, and external rotation were used to examine fatigue resistance on scapular kinematics (Figure 1A). Additionally, we calculated the autocorrelation of the arm elevation angle tracking and horizontal adduction to investigate the statistical stationarity of movement (Figure 1B).

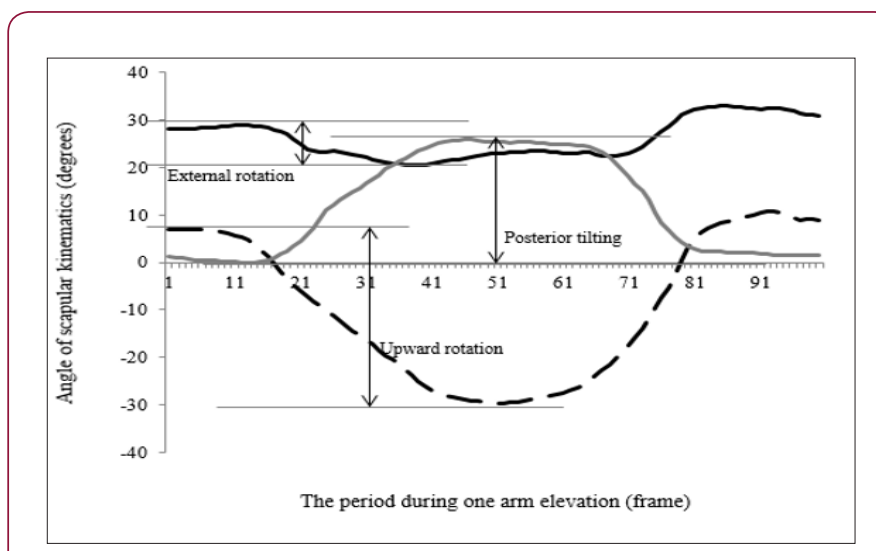

Figure 1A: Scapula kinematics during one arm elevation. Example of the scapula kinematics during one arm elevation. The dashed line represents upward rotation, the gray line represents posterior tilting, and the black line represents external rotation.

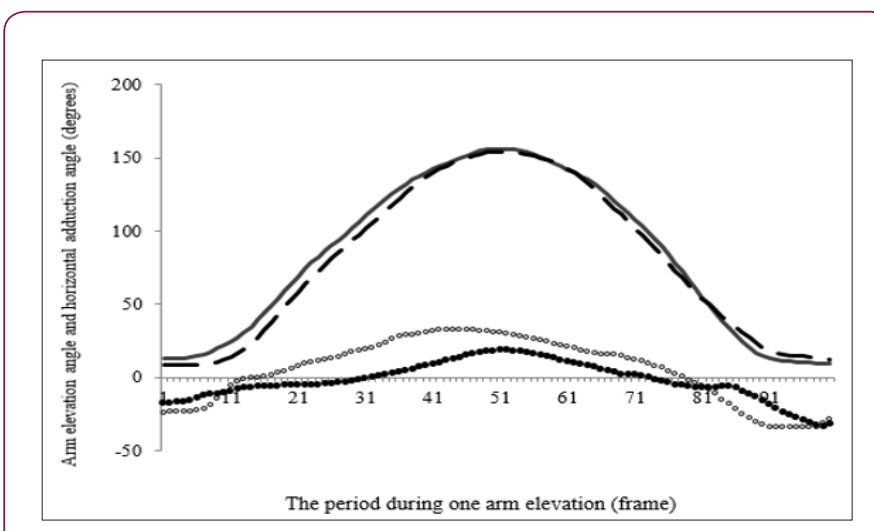

Figure 1B: Tracking of the arm elevation angle and horizontal adduction angle. An example of the tracking of the arm elevation angle and horizontal adduction angle. The black line represents the arm elevation angle at $0 \%$ and the dashed line represents at $25 \%$. The closed circle represents the horizontal adduction angle at $0 \%$ and the open circle represents at $25 \%$. We calculated each autocorrelation from the data of 100 tracks. 
SPSS software for Windows version 24.0 (IBM, Armonk, NY, USA) was used for data analysis. The effects of the conditions $(0 \%$, $25 \%, 50 \%, 75 \%, 100 \%$ ) on scapular upward rotation, posterior tilting, and external rotation were analyzed using a one-way repeated measure analysis of variance (ANOVA). When a significant main effect of the condition was observed, the post-hoc Bonferroni test was used to determine the significant differences. The level of statistical significance was set at $\mathrm{p}<0.05$.

\section{Results}

The maximum shoulder abduction force was $15.6 \pm 2.3 \mathrm{~kg}(13.5$ - $19.3 \mathrm{~kg}$ ), and the dumbbell weight held by participants was $2.0 \pm$ $0.5 \mathrm{~kg}(1.5-3.0 \mathrm{~kg})$. The iteration count of arm elevation was $25.2 \pm$ 10.2 times ( 10 - 38 times). Table 1 shows the autocorrelation of the arm elevation angle tracking and the horizontal adduction during repeated arm elevation.

Table 1: Autocorrelation with the first elevation of the arm elevation and the horizontal adduction.

\begin{tabular}{|c|c|c|c|c|}
\hline & $\mathbf{2 5 \%}$ & $\mathbf{5 0 \%}$ & $\mathbf{7 5 \%}$ & $\mathbf{1 0 0 \%}$ \\
\hline $\begin{array}{c}\text { Arm } \\
\text { elevation }\end{array}$ & $\begin{array}{c}0.95 \\
(0.80-0.99)\end{array}$ & $\begin{array}{c}0.92 \\
(0.69-0.99)\end{array}$ & $\begin{array}{c}0.91 \\
(0.70-0.98)\end{array}$ & $\begin{array}{c}0.90 \\
(0.64-0.98)\end{array}$ \\
\hline $\begin{array}{c}\text { Horizontal } \\
\text { adduction }\end{array}$ & $\begin{array}{c}0.69 \\
(0.15-0.98)\end{array}$ & $\begin{array}{c}0.60 \\
(0.31-0.91)\end{array}$ & $\begin{array}{c}0.64 \\
(0.39-0.91)\end{array}$ & $\begin{array}{c}0.56 \\
(0.18-0.93)\end{array}$ \\
\hline
\end{tabular}

mean (min - max)

The upward rotation angles were as follows: $0 \%, 34.8 \pm 5.7^{\circ}$; $25 \%, 36.2 \pm 7.8^{\circ} ; 50 \%, 37.0 \pm 5.5^{\circ} ; 75 \%, 37.1 \pm 6.1^{\circ} ; 100 \%, 38.1$ $\pm 4.7^{\circ}$. The results from the ANOVA tests indicated a significant effect of the condition on upward rotation. Additionally, there was a significant difference in the angle between the $100 \%$ and $0 \%$ iteration count $(\mathrm{p}=0.014)$ (Figure 2A).

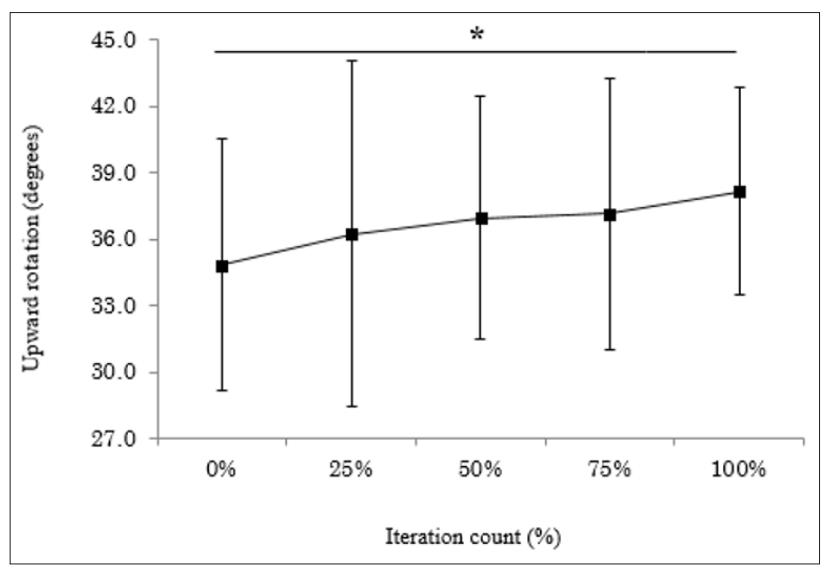

Figure 2A: Scapular upward rotation. Post-hoc analysis revealed the upward rotation angle to significantly increase at the $100 \%$ compared to $0 \%\left({ }^{*} \mathrm{p}<0.05\right)$.

The posterior tilting angles were as follows: $0 \%, 21.6 \pm 7.6^{\circ}$; $25 \%, 22.1 \pm 6.2^{\circ} ; 50 \%, 20.6 \pm 6.3^{\circ} ; 75 \%, 17.8 \pm 8.0^{\circ} ; 100 \%, 17.7$ $\pm 7.8^{\circ}$. The results from the ANOVA tests indicated a significant effect of the condition on posterior tilting. Additionally, post-hoc analysis revealed the angle to significantly decrease at the $75 \%$ and $100 \%$ iteration counts compared to $0 \%$ iteration counts $(\mathrm{p}=0.021)$ (Figure 2B).

The external rotation angles were as follows: $0 \%, 8.8 \pm 4.8^{\circ}$; $25 \%, 7.3 \pm 2.8^{\circ} ; 50 \%, 8.9 \pm 2.9^{\circ} ; 75 \%, 9.9 \pm 4.0^{\circ} ; 100 \%, 11.4 \pm$ $4.0^{\circ}$. The results from the ANOVA tests indicated no effect of the condition on external rotation (Figure $2 \mathrm{C}$ ).

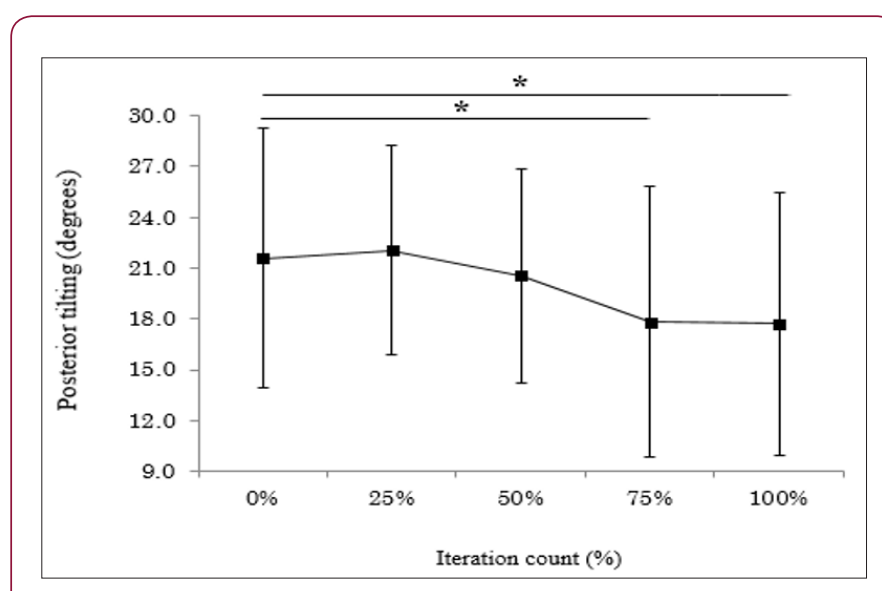

Figure 2B: Scapular posterior tilting. Post-hoc analysis revealed the posterior tilting angle to significantly decrease at the $75 \%$ and $100 \%$ compared to $0 \%\left({ }^{*} \mathrm{p}<0.05\right)$.

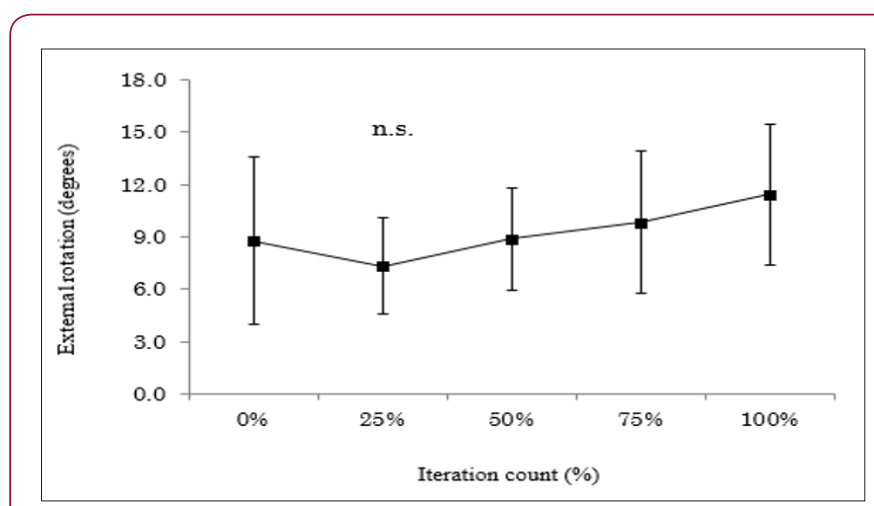

Figure 2C: Scapular external rotation. The results from the ANOVA tests indicated no effect of the condition on external rotation (non-significant [n.s.]).

\section{Discussion}

In the present study, we investigated the effect of fatigue resistance on the scapula kinematics by analyzing the kinematics during repeated arm elevation. Our results showed that the degree of shoulder fatigue contributed to the scapular kinematics. The most important findings of this study were that the angle of upward rotation was maintained until $75 \%$, with an increase observed at $100 \%$, and the angle of posterior tilting was maintained until $50 \%$, with a decrease observed after $75 \%$. 
Generally, there are many reports suggesting that muscle fatigue affects the muscle activation pattern and scapula kinematics [9,10,16-19]. Szucs et al. [17] showed that higher upper trapezius activation may be compensatory for fatigue in the other shoulder muscles. Joshi et al. [19] investigated the influence of externalrotation fatigue and showed increased scapula upward rotation and decreased lower trapezius activity. However, no researchers have previously measured fatigue resistance, which is how much fatigue leads to scapular dyskinesia. Our results showed that the scapula upward rotation at $100 \%$ significantly increased compared to the rotation at $0 \%$. Moreover, it was confirmed by a high autocorrelation of 0.90-0.95 that participants could perform unified arm elevation. It was suggested that muscle fatigue by repeated arm elevation increased the upward rotation, which was maintained until complete exhaustion.

Scapular posterior tilting was maintained until $50 \%$ and a decrease was observed after 75\%. Posterior tilting during arm elevation plays an important role in extending the acromiohumeral distance; a decrease results in the risk of impingement syndrome [20,21]. Lukasiewicz et al. [22] reported subjects with impingement demonstrated a significantly lower posterior tilting angle using a 3-dimensional electromechanical digitizer, suggesting that altered posterior tilting may be an important aspect of the scapular kinematics analysis. However, the evaluation of scapular kinematics is generally carried out by measuring the upper rotation or scapula-spine distance; it is difficult to evaluate alterations in the posterior tilting angle in the clinic [23]. The decrease in the posterior tilting angle was observed before the increase in the upward rotation angle; the decrease in the posterior tilting angle appears when it exceeds $50 \%$. These results may help to keep stress under the surface of the acromion by limiting within $50 \%$ of the iteration count.

No differences were observed in the angle of scapular external rotation during the fatigue task. These findings on the angle of external rotation were not in agreement with those of the study by Chopp et al. [11] and Ebaugh et al. [24], who reported an increase in scapular external rotation. On the other hand, Tsai et al. [25] reported a decrease in the scapular external rotation postfatigue. In this study, we controlled the elevation plane by visual confirmation during the fatigue task; however, autocorrelation of the horizontal adduction tracking was $0.56-0.69$, meaning the elevation may not be in the same plane. These contrasting results concerning the scapular external rotation angle may be due to difficulty in controlling the elevation plane.

The present study had several limitations. First, our participant sample included young adults who were not athletes; when analyzing the repeated arm elevation, the tendency of scapula kinematics might be different depending on the different sports performed by athletes. Second, we did not perform electromyography during the fatigue protocol, and it was unknown which muscles affected the scapula kinematics. In future studies, the scapula kinematics of more complicated movements, such as the throwing motion, should be investigated. Furthermore, the muscles affecting shoulder kinematics should be clarified. Muscle fatigue by repeated arm elevation resulted in an increased the angle of upward rotation, which was maintained until just before complete exhaustion. A decrease in the posterior tilting angle was observed before (exceeding $50 \%$ of the iteration count) the increase in the upward rotation angle. It is suggested that limiting the posterior tilting angle within $50 \%$ of the iteration count might be important to prevent scapular dyskinesia caused by muscle fatigue.

\section{Acknowledgement}

The authors would like to thank those who participated in the study.

\section{References}

1. Van Andel C, Van Hutten K, Eversdijk M, Veeger D, Harlaar J (2009) Recording scapular motion using an acromion marker cluster. Gait Posture 29(1): 123-128.

2. Lo YPC, Hsu YCS, Chan KM (1990) Epidemiology of shoulder impingement in upper arm sports events. Br J Sports Med 24(3): 173-177.

3. Burn MB, McCulloch PC, Lintner DM, Liberman SR, Harris JD (2016) Prevalence of scapular dyskinesis in overhead and nonoverhead athletes. Orthop J Sports Med 4(2): 1-8.

4. Kibler WB, McMullen J (2003) Scapular dyskinesis and its relation to shoulder pain. J Am Acad Orthop Surg 11(2): 142-151.

5. Willmore EG, Smith MJ (2016) Scapular dyskinesia: Evolution towards a systems-based approach. Shoulder Elbow 8(1): 61-70.

6. Sanchez HM, Sanchez EG, Tavares LI (2016) Association between scapular dyskinesia and shoulder pain in young adults. Acta Ortop Bras 24(5): 243-248.

7. Huang TS, Huang CY, Ou HL, Lin JJ (2016) Scapular dyskinesis: Patterns, functional disability and associated factors in people with shoulder disorders. Man Ther 26: 165-171.

8. Kibler WB, Sciascia A (2016) The role of the scapula in preventing and treating shoulder instability. Knee Surg Sports Traumatol Arthrosc 24(2): 390-397.

9. Ebaugh DD, McClure PW, Karduna AR (2006) Scapulothoracic and glenohumeral kinematics following an external rotation fatigue protocol. J Orthop Sports Phys Ther 36(8): 557-571.

10. Maenhout A, Dhooge F, Van Herzeele M, Palmans T, Cools A (2015) Acromiohumeral distance and 3-dimensional scapular position change after overhead muscle fatigue. J Athl Train 50(3): 281-288.

11. Chopp JN, Fischer SL, Dickerson CR (2011) The specificity of fatiguing protocols affects scapular orientation: Implications for subacromial impingement. Clin Biomech 26(1): 40-45.

12. Chopp JN, O’Neill JM, Hurley K, Dickerson CR (2010) Superior humeral head migration occurs following a protocol designed to fatigue the rotator cuff: A radiographic analysis. J Shoulder Elbow Surg 19(8): 11371144.

13. Tse CT, McDonald AC, Keir PJ (2016) Adaptations to isolated shoulder fatigue during simulated repetitive work. Part I: Fatigue. J Electromyogr Kinesiol 29: 34-41.

14. Cadogan A, Laslett M, Hing W, McNair P, Williams M (2011) Reliability of a new hand-held dynamometer in measuring shoulder range of motion and strength. Man Ther 16(1): 97-101.

15. Wu G, Van Helm FC, Veeger HE, Makhsous M, Van Roy P, et al. (2005) ISB recommendation on definitions of joint coordinate systems of various joints for the reporting of human joint motion-Part II: shoulder, elbow, wrist and hand. J Biomech 38(5): 981-992.

16. Cools AM, Witvrouw EE, De Clercq GA, Danneels LA, Willems TM, et al. (2002) Scapular muscle recruitment pattern: Electromyographic 
response of the trapezius muscle to sudden shoulder movement before and after a fatiguing exercise. J Orthop Sports Phys Ther 32(5): 221-229.

17. Szucs K, Navalgund A, Borstad JD (2009) Scapular muscle activation and co-activation following a fatigue task. Med Biol Eng Comput 47(5):487495.

18. McQuade KJ, Dawson J, Smidt GL (1998) Scapulothoracic muscle fatigue associated with alterations in scapulohumeral rhythm kinematics during maximum resistive shoulder elevation. J Orthop Sports Phys Ther 28(2): 74-80.

19. Joshi M, Thigpen CA, Bunn K, Karas SG, Padua DA (2011) Shoulder external rotation fatigue and scapular muscle activation and kinematics in overhead athletes. J Athl Train 46(4): 349-357.

20. Endo K, Ikata T, Katoh S, Takeda Y (2001) Radiographic assessment of scapular rotational tilt in chronic shoulder impingement syndrome. J Orthop 6(1): 3-10.

ISSN: 2574-1241

DOI: 10.26717/BJSTR.2018.10.002022

Hitoshi Koda. Biomed J Sci \& Tech Res

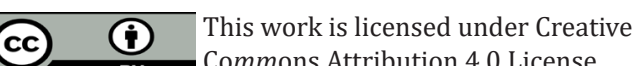

Submission Link: https://biomedres.us/submit-manuscript.php
21. Ludewig PM, Cook TM (2000) Alterations in shoulder kinematics and associated muscle activity in people with symptoms of shoulder impingement. Phys Ther 80(3): 276-291.

22. Lukasiewicz AC, McClure P, Michener L, Pratt N, Sennett B (1999) Comparison of 3-dimensional scapular position and orientation between subjects with and without shoulder impingement. J Orthop Sports Phys Ther 29(10): 574-583.

23. Kibler WB, Sciascia A (2010) Current concepts: Scapular dyskinesis. Br J Sports Med 44(5): 300-305.

24. Ebaugh DD, McClure PW, Karduna AR (2006) Effects of shoulder muscle fatigue caused by repetitive overhead activities on scapulothoracic and glenohumeral kinematics. J Electromyogr Kinesiol 16(3): 224-235.

25. Tsai NT, McClure PW, Karduna AR (2003) Effects of muscle fatigue on 3-dimensional scapular kinematics. Arch Phys Med Rehabil 84(7): 10001005.

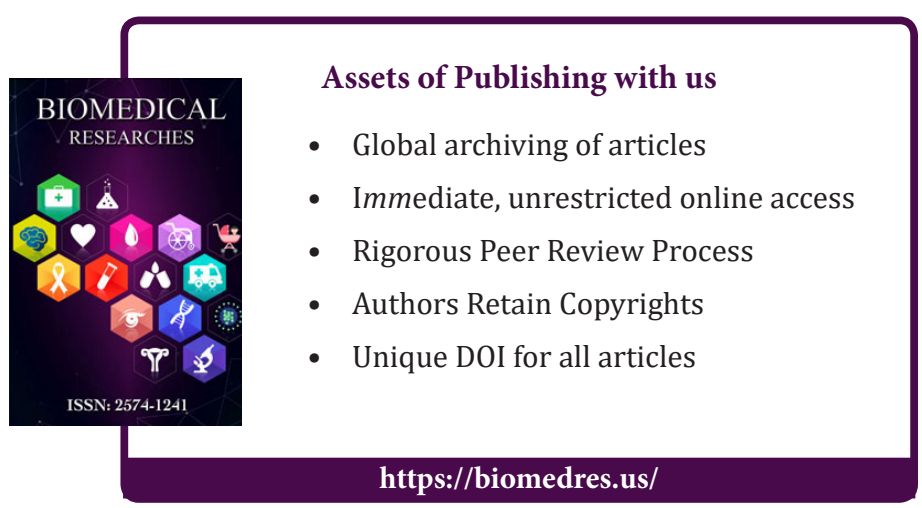

